\title{
Poster: Unveiling the Missed 4.5G Performance In the Wild
}

\author{
Kai Ling, Haotian Deng, Junpeng Guo, Chunyi Peng \\ Department of Computer Science, Purdue University \\ [ling59,deng164,guo567,chunyi]@purdue.edu
}

\begin{abstract}
In this poster, we present our efforts to extend our recent study [1]. In [1], we used a city-scale measurement to unveil an important but unexplored performance issue in operational $4.5 \mathrm{G}$ networks: significant performance miss indeed happens and happens quite frequently in the wild. In this poster, we present our longitudinal study to extend our reality check to a larger scale. In particular, we investigate the impact of the dataset size, and discuss challenges for a large-scale study with high precision confidence.
\end{abstract}

\section{CCS CONCEPTS}

- Networks $\rightarrow$ Mobile networks; Network manageability.

\section{ACM Reference Format:}

Kai Ling, Haotian Deng, Junpeng Guo, Chunyi Peng. 2020. Poster: Unveiling the Missed 4.5G Performance In the Wild. In The 21st International Workshop on Mobile Computing Systems and Applications (HotMobile '20), March 3-4, 2020, Austin, TX, USA. ACM, New York, NY, USA, 1 page. https: //doi.org/10.1145/3376897.3379170

\section{INTRODUCTION}

In our recent work [1], we have presented the arguably first study to unveil and understand the missed performance in the wild. The missed performance is defined as the gap between what a mobile device actually gets in reality and what it could have possibly got at best, given the same operational network. We have conducted a cityscale measurements in the small city of West Lafayette, IN $(6 \mathrm{~km} \times$ $6.7 \mathrm{~km}$ ) over all four top US carriers (AT\&T, Verizon, T-Mobile and Sprint). Our results show that significant performance miss indeed happens and happens frequently. More details can be found in [1].

In this poster, we focus on extending this study to a larger-scale reality check. We aim to measure how the performance gaps vary at more places and over a longer time. We need to conduct this measurement efficiently while ensuring high confidence of precision. To this end, we must tackle one problem which remains unaddressed in [1]. How do we know that there are sufficient observations to quantify the missed performance in reality? We take a longitudinal study to investigate how the results vary with more samples over time. We identify one new challenge but also one positive result for reality-check at scale.

Permission to make digital or hard copies of part or all of this work for personal or classroom use is granted without fee provided that copies are not made or distributed for profit or commercial advantage and that copies bear this notice and the full citation on the first page. Copyrights for third-party components of this work must be honored. For all other uses, contact the Owner/Author(s).

HotMobile '20, March 3-4, 2020, Austin, TX, USA

(C) 2020 Copyright is held by the owner/author(s).

ACM ISBN 978-1-4503-7116-2/20/03.

https://doi.org/10.1145/3376897.3379170
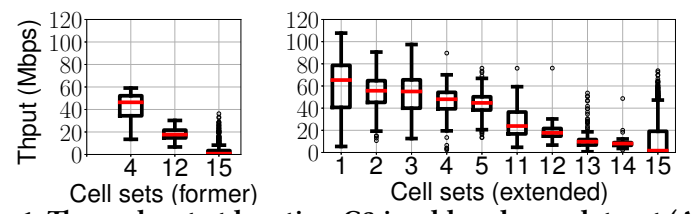

Figure 1: Throughput at location $C 3$ in old and new dataset (AT\&T).

\section{EXTENDED WORK AND NEW INSIGHTS}

We take a longitudinal study. We run many more repeated experiments over longer periods of time to compare how the results vary over the former and extended datasets. In principle, there are two cases. First, the findings are similar, which indicates that there are sufficient samples in our former study; Second, the findings vary in the new study with a larger dataset. We have conducted more experiments (56hrs, $1,434 \mathrm{~km}$ ) to examine the impact of increasing data samples on the serving cell sets and their observed performance. We have made two new findings.

First, more serving cell sets are observed at some places in our extended work. For instance, at location C3, we observed only 3 sets in [1] but 15 sets with many more runs (Figure 1). In another example over one specific driving route (approximately $1 \mathrm{~km}$ ), we have seen that the total number of unique serving cell sets grows from 93 to 156 when we run more experiments for four extra hours, which doubles the size of our former study. This raises a practical challenge for a larger-scale study. To capture the precise performance in reality, we have to run many more experiments over a much longer period at each test location. We should monitor how the results vary with more runs over time and ensure sufficient samples until they converge. In our current study, we find that we need to run at least several hours ( $>5$ hours) at each location with dense cell development ( $>10$ cells).

Second, data performance of the serving cell set is relatively stable over time. This makes possible to approximate the miss performance (actually its lower bound) with no need of running many experiments to capture all the serving cell sets. Take the example at C3. Sets 4, 12 and 15 are observed in both former and extended studies. Their observed data speeds are quite close. For instance, the median speed by set 4 is $46.3 \mathrm{Mbps}$ in [1] and slightly grows to $48.2 \mathrm{Mbps}$. We have similar observations by set 12 and set 15 . We note distinct performance variance by set 15 . This is because some good performance (up to 20Mbps) is observed with more runs while its median speed still remains low at most runs. We have seen similar results at most locations. This implies that our former study is still able to assess the performance gap (actually, its lower bound), even though it failed to capture all the serving cell sets.

\section{REFERENCES}

[1] Haotian Deng, Kai Ling, Junpeng Guo, and Chunyi Peng. 2020. Unveiling the Missed 4.5G Performance In the Wild. In Proceedings of the 21st International Workshop on Mobile Computing Systems and Applications (HotMobile'20). 